CASE REPORT

\title{
Possible Anaphylactic Reaction Due to Pulmonary Hydatid Cyst Rupture Following Blunt Chest Trauma: A Case Report and Review of the Literature
}

\author{
Mehmet Topuzlar, MD; Cenker Eken, MD; Birsen Ozkurt, MD; Feras Khan, MD \\ From the Gulhane Military Medical Academy, Department of Emergency Medicine, Ankara, Turkey (Drs Topuzlar, Eken, and Ozkurt); and \\ the State University of New York-Downstate Medical Center/Kings County Hospital Center, Department of Emergency Medicine and Internal \\ Medicine, Brooklyn, NY (Dr Khan)
}

\begin{abstract}
A hydatid cyst is a parasitic disease caused by the tapeworm Echinococcus granulosus. It is endemic in many areas, including New Zealand, Australia, and the Mediterranean region. Pulmonary hydatid disease can be diagnosed incidentally in asymptomatic patients or may cause symptoms such as cough, chest pain, dyspnea, fever, and hemoptysis both in patients with ruptured and nonruptured cysts. Anaphylactic reaction is a rare presentation of pulmonary hydatid cyst disease. In this case report, we report an unusual anaphylactic reaction following pulmonary hydatid cyst rupture secondary to blunt chest trauma.
\end{abstract}

Key words: pulmonary hydatid cyst, blunt chest trauma, anaphylactic reaction, Echinococcus granulosus

\section{Introduction}

A hydatid cyst is a parasitic disease caused by the tapeworm Echinococcus granulosus. The tapeworm stage takes place in the intestine of a carnivore (usually a dog), the definitive host. The tapeworm eggs are found in the feces of an infected carnivore and are ingested by an herbivore (eg, sheep), the intermediate host. The tapeworm eggs hatch in the intestine of the herbivore and reach the liver via the portal vein where they develop into hydatid cysts. The eggs also reach other organs via the lymphatic system. Echinococcus infection occurs in humans following inadvertent ingestion of tapeworm eggs. ${ }^{1}$

Hydatid disease is frequently encountered in endemic areas, such as several Mediterranean countries, New Zealand, Australia, North America, South America, Central America and Asia. ${ }^{2-5}$ The liver is the most frequently affected organ, followed by the lungs (55\% and $40.4 \%$, respectively). Other organs such as the spleen, kidney, and bone are less commonly affected by hydatid disease. $^{6}$

Corresponding author: Cenker Eken, MD, Gulhane Military Medical Academy Department of Emergency Medicine, Ankara, Turkey (e-mail: cenkereken@akdeniz.edu.tr).
Hydatid cyst disease has silent and gradual development during the first few years until symptoms are caused by its space-occupying mass effect, mechanical obstruction, rupture, or secondary allergic reactions. In the present case, we report an unusual presentation of pulmonary hydatid cyst rupture following blunt chest trauma that presented as a likely anaphylactic reaction initially misdiagnosed as a hemothorax.

\section{Case}

An 18-year-old male presented to the emergency department with an altered mental status and shortness of breath. He was involved in a fistfight in which he suffered blunt trauma when he was punched in the upper back over the posterior hemithorax. He had no significant past medical history. In the primary survey, his airway was intact but he was tachypenic and had a shallow respiratory pattern. His carotid pulse was thready. He was not oriented to person, place, or time, and he was agitated. His blood pressure was not detectable and his pulse rate was 80 beats per minute. He had an oxygen saturation of $56 \%$ by pulse oximeter. In the secondary survey, he had both peripheral cyanosis in his nail beds and central cyanosis in his lips. His breath sounds were 


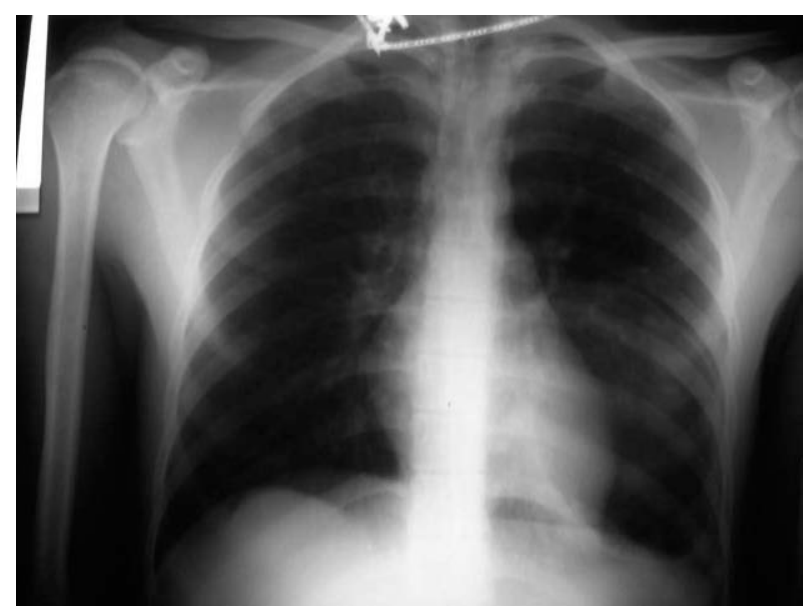

Figure 1. The chest x-ray with a dense opacity occupying the lower part of the left lung.

coarse bilaterally and diminished in the left hemithorax. A $10-\mathrm{mL} /$ minute oxygen nonrebreather facemask was placed on the patient and boluses of crystalloid infusion were started using 16-gauge and 20-gauge peripheral intravenous lines. We suspected a hemothorax and we planned to intubate the patient and place a chest tube. However, a bedside ultrasonography (US) scan and a portable chest $\mathrm{x}$-ray were performed immediately before any procedures were done. The chest $\mathrm{x}$-ray revealed a dense opacity occupying the lower part of the left lung, the right diaphragm was elevated, and there was a double contour to the right hemidiaphragm (Figure 1). There was no free fluid seen on the focused abdominal sonography in trauma (FAST) US exam. The bedside US did reveal 3 anechoic cystic masses approximately $8-10 \mathrm{~cm}$ in diameter in the liver. We made a presumptive diagnosis of hydatid cyst disease, and thought that pulmo-

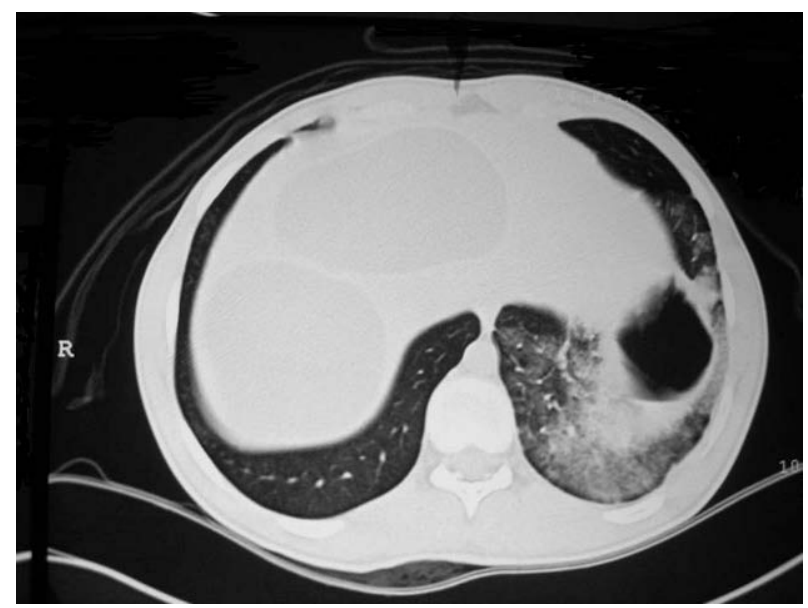

Figure 2. The air in the cavity and pericystic consolidation suggesting fluid leakage or an inflammatory reaction.

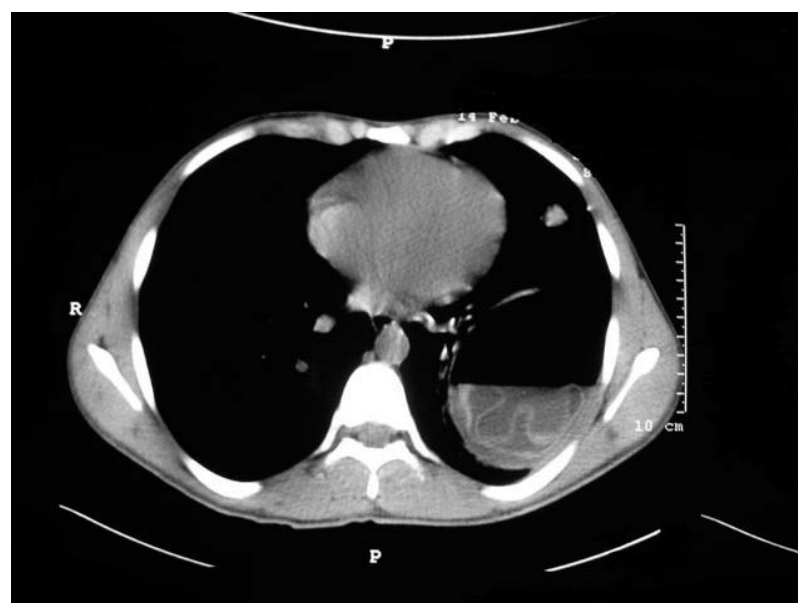

Figure 3. The floating detached membrane of the hydatid cyst.

nary cyst rupture might be causing his symptoms. The patient's mental status and vital signs improved dramatically after the crystalloid infusion and oxygen support were started, and we decided against intubation at that time. The patient's blood pressure increased to $140 / 80$ $\mathrm{mm} / \mathrm{Hg}$, and oxygen saturation to $93 \%$ by pulse oximeter. He was now oriented and no longer agitated. Thoracic and abdominal computerized tomography (CT) was done. The thoracoabdominal CT revealed a ruptured pulmonary hydatid cyst, which had opened into the pulmonary parenchyma and bronchial tree, in addition to nonruptured hydatid disease of the liver. The pulmonary hydatid cyst with air inside and the disrupted capsule are displayed in Figures 2 and 3. The hydatid cysts in the liver can be seen in Figure 4. The patient developed hemoptysis following the $\mathrm{CT}$ procedure. The patient was then admitted to the thoracic surgery ward where he subsequently had 3 further episodes similar to his initial

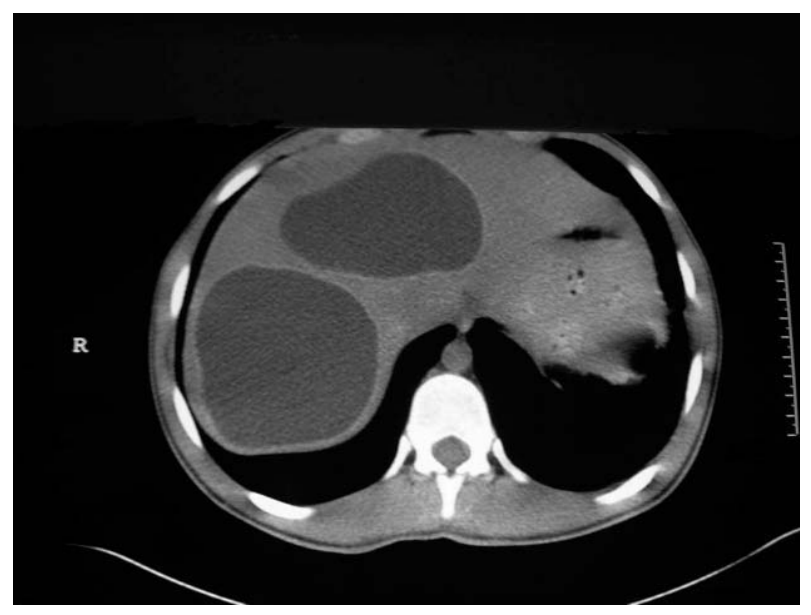

Figure 4. The hydatid cysts in the liver. 


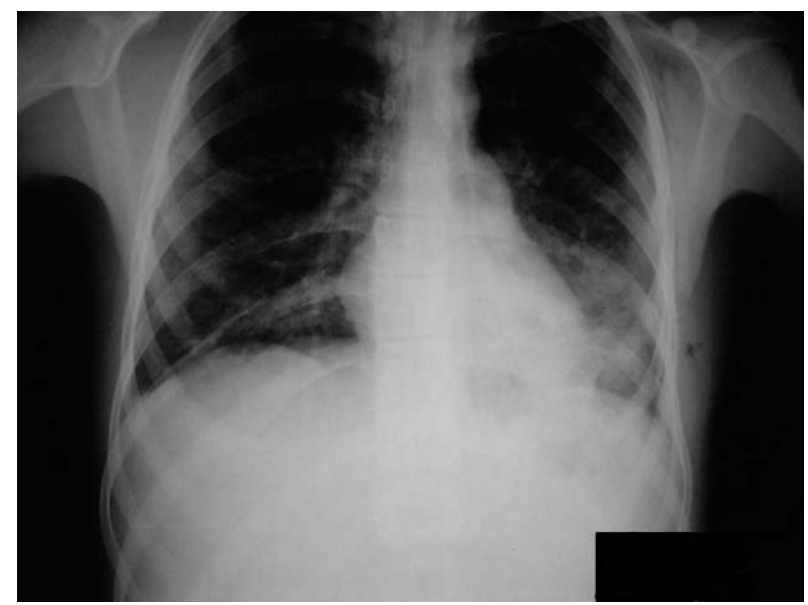

Figure 5. The 1-month follow-up chest x-ray.

presenting condition. He eventually recovered with supportive care. The patient underwent an operation with hydatid cyst excision. He was treated with albendazole $200 \mathrm{mg}$ orally twice daily, and had no complaints when he returned for his 1-month follow-up. His 1-month follow-up chest x-ray is displayed in Figure 5.

\section{Discussion}

Hydatid disease is endemic in many countries, with a considerable morbidity and mortality rate. Hydatid cysts have 2 layers, outer and inner, with a liquid content and antigenic elements that can cause anaphylactic reactions. $^{7}$

A hydatid cyst can develop anywhere in the lung, but it more often settles in the right lower lobe. In 3 of 4 cases found in the literature, patients presented with single cysts. ${ }^{8}$ Uncomplicated single cysts can cause atelectasis due to compression of the lung parenchyma and the inflammatory response surrounding the cyst. The cyst can also occlude neighboring bronchi causing a distal lesion or can completely destroy a whole lobe. ${ }^{7}$

One third of patients with pulmonary hydatidosis present with ruptured cysts. ${ }^{8}$ The cyst may rupture into a bronchus, releasing clear and salty fluid that contains the parasitic membrane and hydatid vesicles (hydatid vomica). The vomica, with or without hemoptysis, can cause asphyxia, anaphylactic shock, secondary hydatid spread, acute respiratory failure, massive hemoptysis, or even cardiovascular collapse. Hydatid cysts rupturing into a bronchial lumen can either be totally eliminated by the lymphatic system or leave residual cavities that may become infected, resulting in an unfavorable outcome.

The initial growth of a single cyst is usually asymptomatic. Hydatid cysts can be diagnosed incidentally during radiologic evaluation for another reason. Various symptoms, such as chest pain, hemoptysis, dyspnea, and fever, can be seen with hydatid cysts. Kuzucu et $\mathrm{al}^{9}$ reported chest pain $(50 \%)$, cough $(26.5 \%)$, dyspnea $(17.6 \%)$, and fever $(11.8 \%)$ as the most common presenting symptoms in patients with intact pulmonary hydatid cysts, and $26.5 \%$ of patients were asymptomatic. However, in ruptured pulmonary cysts, chest pain (48.5\%), cough $(45.5 \%)$, dyspnea $(42.4 \%)$, hemoptysis (33.3\%), fever (36.4\%), and sputum production (33.3\%) were the most common presenting symptoms. Only $3 \%$ of the patients with ruptured pulmonary hydatid cysts were asymptomatic. ${ }^{9}$ Darwish ${ }^{10}$ reported cough as the primary symptom (54\%) in 168 symptomatic patients with pulmonary hydatid cysts. Chest pain (36\%), dyspnea $(25 \%)$, hemoptysis (19\%), hydatidemesis $(10 \%)$, infectious symptoms (10\%), pleuritis $(5 \%)$, and pneumothorax (3\%) were the other presenting symptoms, and $18 \%$ of the patients were asymptomatic. ${ }^{10}$

Anaphylactic reactions due to rupture of hydatid cysts following blunt trauma are very rare. Traumatic rupture of hepatic and splenic hydatid cysts resulting in anaphylactic reaction has been reported in the literature, but we could find no reports of such reactions following blunt chest trauma. In the present case, despite the absence of other symptoms seen in anaphylaxis, such as rash, edema, and bronchospasm, the hemodynamic and respiratory compromise during the emergency department presentation of the patient were felt to be due to anaphylactic reaction, as were the latter 3 similar episodes while on the thoracic surgery ward. The actual diagnosis in our case remains unclear, and the lack of administering antihistamines, steroids, or adrenaline because of the dramatic improvement of the patient was a deficiency of our management. The latter episodes may have been prevented by this therapy. Fanne et al ${ }^{11}$ also reported a similar case with a spontaneous rupture of a pulmonary hydatid cyst that resulted in shock with hypotension and acute respiratory distress syndrome despite the lack of rash and edema.

Hydatid cysts can be diagnosed using direct radiography, US, CT, or magnetic resonance imaging (MRI). A ruptured single cyst is not usually seen as a homogenous opacity with clear edges. The edges of the cyst may be blurred indicating inflammation or pericystic pneumonia. Atelectasis or pneumonitis may indicate a distal lesion, or an opacity involving an entire lobe may indicate total occlusion. After rupture into a bronchial lumen, images may vary. The parasitic membrane partially detached from the adventitia, drops and floats in the residual fluid. The endocyst membrane floating on top of the remaining fluid is called the water lily sign. ${ }^{12}$ The floating detached membrane can be seen in Figure 3 in the present case. In the present case, there was also 
air in the cavity and pericystic consolidation, as seen in Figure 2, suggesting fluid leakage or an inflammatory reaction. Air fluid levels, air bubbles, and cavitation may also be seen in a ruptured cyst. ${ }^{13-15} \mathrm{~A}$ cyst that ruptures into the pleural cavity can present as a hydrothorax or hydropneumothorax. However, there was no sign of hydropneumothorax both in the chest X-ray and CT in the present case. Ultrasonography, CT, and MRI may also be used as alternatives to plain radiography. Although CT has a sensitivity of $100 \%$ in demonstrating cyst rupture, US is more practical and inexpensive. ${ }^{16-18}$ However, there is not enough research evaluating the usefulness of US in pulmonary hydatid cyst. El Fortia et al ${ }^{19}$ reported the specificity of US in diagnosing pulmonary hydatid cysts using the wall sign, a double-layered border in univesicular and double-layered internal septum in multivesicular pulmonary echinococcal cysts. The specificity was $66 \%$ and $100 \%$ in the univesicular and multivesicular cysts, respectively. ${ }^{19}$ Plain chest $\mathrm{x}$-ray detected only 57 of 79 pulmonary cysts detected by CT in the study by Erdem et al. ${ }^{20}$ Magnetic resonance imaging can also be used in the diagnosis of pulmonary hydatid disease. ${ }^{21,22}$

Laboratory testing should be used either in highly suspicious cases or for the postoperative follow-up of pulmonary hydatid cyst disease. Both qualitative (immunoelectrophoresis) and quantitative enzyme-linked immunosorbent assay (ELISA) tests are available for hydatid disease. The most sensitive technique in detecting pulmonary hydatidosis is immunoglobulin G ELISA, with a sensitivity of $83.5 \%$. Antibody production is elevated during the first 4 to 6 weeks after surgical intervention, followed by a decrease during the 12 to 18 subsequent months. In patients with a recurrence before 2 years, antibody production remains similar to the preoperative levels. ${ }^{23,24}$ There are also some conflicting results in the literature regarding ELISA in the detection of pulmonary hydatid cysts. Force et $\mathrm{al}^{25}$ reported a lower sensitivity (81\%) despite combination of IgG ELISA, indirect hemagglutination, and IgA ELISA.

The main treatment of a ruptured pulmonary hydatid cyst is surgery. Operative morbidity is higher in complicated pulmonary hydatid cysts than intact ones. ${ }^{9}$ The most frequent postoperative morbidity of a complicated pulmonary hydatid cyst is prolonged air leakage $(15.2 \%)$, empyema $(9.1 \%)$, and atelectasis $(9.1 \%)$. The complication rate is much lower in patients with intact pulmonary hydatid cysts. Prolonged air leak (2.9\%), pleural effusion (2.9\%), and wound infection (2.9\%) are the complications seen during the postoperative period in patients with intact pulmonary hydatid cysts. ${ }^{9}$ Operative mortality generally does not exceed $1 \%$ to $2 \%$. Medical treatment of pulmonary hydatid cyst, using al- bendazole or mebendazole, is used as a complement to surgical treatment to avoid recurrence. It is applied preoperatively to prevent the consequences of possible rupture of the cysts during surgery and postoperatively as adjuvant therapy of the cysts that may have ruptured during the operation. Keshmiri et $\mathrm{al}^{26}$ showed that albendazole is superior to placebo in terminating pulmonary hydatid cysts, but only 16 of 124 cysts in a 6-month period were treated successfully. Dogru et $\mathrm{al}^{27}$ reported a cure rate of $36.3 \%$ with medical treatment in children. Medical therapy was particularly effective in cysts with a small size. The mean diameter of successfully treated cysts was $5.3 \mathrm{~cm}$, whereas cysts that were over $7.3 \mathrm{~cm}$ in size failed medical treatment. Complications due to medical treatment have also been reported in the medical literature. Keramidas et al ${ }^{28}$ reported a complication rate of $30.5 \%$ with medical treatment of pulmonary hydatid disease. Most of these complications were pleural empyemas and lung abscesses. Furthermore, larger cysts lead to higher complication rates. All patients in the Keramidas study had a cyst size of more than $6 \mathrm{~cm}$ in diameter. ${ }^{28}$ Dogan et $\mathrm{al}^{29}$ stated that 4 of 28 patients had to undergo urgent operation owing to massive hemoptysis after receiving mebendazole. Medical treatment should also be used in patients who cannot undergo operation. ${ }^{30}$

In conclusion, pulmonary hydatid cyst disease is still an endemic disease in many countries. Either ruptured or nonruptured cysts may present with varying symptoms. Anaphylaxis due to traumatic rupture of a hydatid cyst is a rare presentation and should be suspected in patients in endemic countries and in immigrants from endemic countries in nonendemic regions.

\section{References}

1. Chadli D. Hydatid disease-continuing serious public health problem: introduction. World J Surgery. 2001;25: $1-3$.

2. Tselentis J, Karpathios T, Fretzayas A, Korkas A, Nicolaidou P, Matsaniotis N. Hydatid disease in asymptomatic young carriers in northern Greece. Am J Trop Med Hyg. 1983;32:1462.

3. Chipponi J, Huguier M. Surgically treated hydatid cysts of the liver in France: epidemiology, diagnostic, and therapeutic procedures-A study of 306 cases [in French]. Gastroenterol Clin Biol. 1986;10:419.

4. Bchir A, Hamdi A, Jemni L, et al. Serological screening for hydatidosis in households of surgical cases in central Tunisia. Ann Trop Med Parasitol. 1988;82:271.

5. Andersen FL. Introduction to cystic echinococcosis and description of cooperative research project in Morocco. In: Andersen FL, Ouhelli H, Kachani M, eds. Compendium on Cystic Echinococcosis in Africa and in Middle Eastern 
Countries with Special Reference to Morocco. Provo, UT: Brigham Young University; 1997:1-17.

6. Hsairi M, Chahed MK, Bchir A, et al. Surgical incidence of hydatidosis in Tunisia 1988-1992 [in French]. Tunis Chir. 1995;4:20.

7. Ramos G, Orduna A, Garcia-Yuste M. Hydatid cyst of the lung: diagnosis and treatment. World J Surg. 2001;25:4657.

8. Aytac A, Yurdakul Y, İkizler C, Olga R, Saylam A. Pulmonary hydatid disease: report of 100 patients. Ann Thorac Surg. 1977;23:145.

9. Kuzucu A, Soysal O, Ozgel M, Yologlu S. Complicated hydatid cysts of the lung: clinical and therapeutic issues. Ann Thorac Surg. 2004;77:1200-1204.

10. Darwish B. Clinical and radiological manifestations of 206 patients with pulmonary hydatidosis over a ten year period. Prim Care Resp J. 2006;15:246-251.

11. Fanne RA, Khamaisi M, Mevorach D, et al. Spontaneous rupture of lung echinococcal cyst causing anaphylactic shock and respiratory distress syndrome. Thorax. 2006;61: 550.

12. Schlanger PM, Schlanger H. Hydatid disease and its roentgen picture. Am J Roentgenol. 1984;60:331-347.

13. Tor M, Ozvaran K, Ersoy Y, et al. Pitfalls in the diagnosis of complicated pulmonary hydatid disease. Resp Med. 2001;95:237-239.

14. Kokturk O, Ozturk C, Diren B, Unsal M, Ayla K. "Air bubble": a new diagnostic CT sign of perforated pulmonary hydatid cyst. Eur Radiol. 1999;9:1321-1323.

15. Zidi A, Ben Miled-Mrad K, Hantous-Zannad S, Fathallah B, Mestiri I, Baccouche I. Computed tomography of complicated pulmonary cyst by rupture in the bronchi. $J R a$ diol. 2007;88:59-64.

16. Erkilic S, Ozsarac C, Kocer NE, et al. Hydatid cyst of the thyroid gland in a child. Int J Pediatr Otorhinolaryngol. 2004;68:369-371.

17. Gunay K, Taviloglu K, Berber E, et al. Traumatic rupture of hydatid cysts: a 12-year experience from an endemic region. J Trauma. 1999;46:164-167.

18. Sezgin O, Altintas E, Saritas U, et al. Hepatic alveolar echinococcosis: clinical and radiological features and en- doscopic management. J Clin Gastroenterol. 2005;39: 160-167.

19. El Fortia M, El Gatit A, Bendaoud M. Ultrasound wallsign in pulmonary echinococcosis (new application). $\mathrm{Ul}$ traschall Med. 2006;27:553-557.

20. Erdem CZ, Erdem LO. Radiological characteristics of pulmonary cyst hydatid disease in children. Less common radiological appearances. Eur J Radiol. 2003;45:123-128.

21. Marani SAD, Canossi GA, Nicoli FA, Alberti GP, Monni SG, Casolo PM. Hydatid disease: MR imaging study. $R a$ diology. 1990;175:701-706.

22. Singh S, Gibikote SV, Sen S, Korula A, Korah IP. Pulmonary hydatid disease mimicking sequestration: differentiation on magnetic resonance imaging. Austuralas $R a$ diol. 1999;43:539-541.

23. Zarzosa MP, Orduña Domingo A, Gutiérrez P, et al. Evaluation of six serological tests in diagnosis and postoperative control of pulmonary hydatid disease patients. Diagn Microbiol Infect Dis. 1999;35:255.

24. Bustamante AJ, García San José I, Quinzaños ER, Tasis A. Hidatidosis (equinococosis): micosis y parasitosis pulmonares II. Patología Aparato Respiratorio. 1993;122:13.

25. Zapatero J, Madrigal L, Lago J, Baschwitz B, Pérez E, Candelas J. Surgical treatment of thoracic hydatidosis: a review of 100 cases. Eur J Cardiothorac Surg. 1989;3: 436.

26. Keshmiri M, Baharvahdat H, Fattahi SH, et al. A placebo controlled study of albendazole in the treatment of pulmonary echinococcosis. Eur Respir J. 1999;14:503-507.

27. Dogru D, Kiper N, Ozcelik U, Yalcin E, Gocmen A. Medical treatment of pulmonary hydatid disease: for which child? Parasitol Int. 2005;54:135-138.

28. Keramidas D, Mavridis G, Souits M, Passalidis A. Medical treatment of pulmonary hydatidosis: complications and surgical treatment. Pediatric Surg Int. 2004;19:774776.

29. Dogan R, Yüksel M, Çetin G, Süzer K, Alp M, Kaya S, et al. Surgical treatment of hydatid cysts of the lung: report on 1055 patients. Thorax. 1989;44:192.

30. Force L, Torres JM, Carillo A, Busca J. Evaluation of eight serological tests in the diagnosis of human echinococcus and follow-up. Clin Infect Dis. 1992;15:473-480. 